Nota científica

\title{
Larvas y estadíos tempranos de desarrollo de crustáceos decápodos de Chile: descripciones publicadas
}

\author{
Ingo S. Wehrtmann*1 y Pedro Báez** \\ *Instituto de Zoología «Ernst Kilian», Universidad Austral de Chile \\ Casilla 567, Valdivia, Chile \\ **Museo Nacional de Historia Natural, Sección Hidrobiología \\ Casilla 787, Santiago, Chile
}

\begin{abstract}
RESUMEN. Las descripciones larvarias de decápodos son esenciales para la comprensión de las estrategias de la historia de vida, relaciones stock-reclutamiento y ecología planctónica. Para facilitar tales estudios, se entrega información concerniente a las descripciones publicadas de los estadíos tempranos de los decápodos que habitan aguas marinas y continentales chilenas. La publicación de los estudios morfológicos sobre larvas de decápodos comenzó en Chile en 1936, y el número de publicaciones mostró un claro aumento entre 1967 y 1973 . Hasta la fecha, se ha descrito el desarrollo larvario completo de un 13,4\% de los decápodos chilenos. El conocimiento concerniente a los estadíos larvarios de los camarones carídeos es extremadamente pobre, si se compara con la información disponible para las especies de anomuros (16\%) y braquiuros (15\%) reportados para Chile. La ausencia de descripciones larvarias para algunas especies comercialmente importantes no solamente dificulta la comprensión de la biología de estos crustáceos, sino también complica el desarrollo de estrategias de manejo adecuado.
\end{abstract}

Palabras claves: morfología larvaria, decápodos marinos y de aguas continentales, lista de especies, Chile.

\section{Larvae and early development stages of decapod crustaceans from Chile: published descriptions}

\begin{abstract}
Larval descriptions of decapod crustaceans are essential for the understanding of life history strategies, stock-recruitment relationships, and plankton ecology. To facilitate such studies, we provide information concerning published descriptions of the early life stages of marine and freshwater decapods inhabiting Chilean waters. Publication of morphological studies on larval decapods started in Chile in 1936, and the number of such publications showed a clear peak between 1967 and 1973. Up to now, the complete larval development of 13.4\% of the Chilean decapods has been described. Knowledge regarding the larval stages of caridean shrimps is extremely poor, while such information is available for $16 \%$ and $15 \%$ of the anomuran and brachyuran species reported from Chile, respectively. The absence of larval descriptions for some commercially important species affect not only the understanding of the biology of these decapods, but also complicates the development of adequate management strategies.
\end{abstract}

Key words: larval morphology, marine and freshwater decapods, species list, Chile.

\section{INTRODUCCION}

Los resultados de varias expediciones nacionales e internacionales (Retamal, 1981; Brattström y Johanssen, 1983) han generado un amplio conocimiento acerca de la composición taxonómica de la fauna carcinológica de Chile. Según Báez (1995), el número total de especies de decápodos reporta- das para Chile asciende a 203. Sin embargo, el catastro todavía no está concluido y se estima el número actual en por lo menos de 250 especies (R. Soto, com. pers.).

En general, la taxonomía de los diferentes estadíos larvales de decápodos es escasamente do- 
cumentada comparándola con estudios similares sobre los adultos (Ingle, 1992). A diferencia de los adultos, las larvas de decápodos pueden ser difíciles de determinar a nivel de especie. Frecuentemente, la asignación de larvas por familia o género es una tarea complicada. Por lo tanto, no sorprende que muchos estudios sobre la presencia de larvas de decápodos en el plancton se refieran a ellas como pertenecientes a categorías taxonómicas de rango superior a especie (para Chile: Palma, 1976, 1980; Palma et al., 1976).

La determinación de larvas de decápodos a nivel de especie juega un rol de gran importancia en estudios relacionados con el ciclo de vida (Jalihal et al., 1993; Anger, 1995). Lo mismo es válido para la clasificación de los decápodos (Rice e Ingle, 1975; Rice, 1980), en la identificación de centros de reproducción (Anger et al., 1994; Wehrtmann, 1994; Wehrtmann y Greve, 1995), en la descripción de ciclos estacionales de la reproducción (Lindley, 1987; Wehrtmann, 1989; Lindley et al., 1993; Palma, 1994; Palma y Arana, 1997) y para establecer la importancia de las larvas como parte de la cadena trófica en la comunidad marina (Morgan, 1990, 1992), por mencionar algunos aspectos de relevancia. El objetivo principal del presente trabajo es entregar un resumen de las descripciones publicadas acerca de decápodos marinos y de aguas continentales presentes en aguas chilenas, que permita facilitar este tipo de estudios.

\section{MATERIALES Y METODOS}

El presente trabajo se refiere exclusivamente a descripciones larvales y de estadíos tempranos de especies de decápodos que forman parte de la carcinofauna chilena. Por lo tanto, la descripción larval de Geryon quinquedens (Perkins, 1973) no está incluida, porque dicho trabajo está basado en larvas de una hembra recolectada en Delaware Bay y corresponde entonces, según la revisión de Manning y Holthuis (1989), a Chaceon quinquedens, conocida para el Atlántico noroeste.

Los autores están concientes que existe más información sobre larvas de decápodos de Chile, incluida en tesis e informes. Sin embargo, debido al difícil acceso a este tipo de literatura, especialmente para investigadores extranjeros, el presente trabajo se restringe sólo a las descripciones publicadas. El número de especies para las Tablas 3 y 4 está basado en un listado de crustáceos decápodos chilenos elaborado por Soto (com. pers.), que presenta la información más actualizada. La sistemática de estas tablas sigue la clasificación sugerida por Bowman y Abele (1982).

\section{RESULTADOS Y DISCUSION}

\section{La historia de las descripciones larvales en Chile}

La primera descripción de un estadío larval correspondiente a un decápodo de aguas chilenas fue publicada por Schwabe (1936). Un año después Gurney (1937) describió el desarrollo larval de Chorismus antarcticus. Con el trabajo de Costlow y Fagetti (1967) se iniciaron en Chile los estudios relativos a la descripción del desarrollo larval en condiciones de laboratorio. Entre 1967 y 1973 se produjo el mayor número de publicaciones sobre este tema (Fig. 1), principalmente gracias a los estudios realizados por Elda Fagetti e Italo Campodonico (ver referencias). Sin duda, este aumento considerable de publicaciones sobre la morfología larvaria de decápodos estuvo relacionado con el desarrollo de técnicas que permitieron mantener larvas y cultivarlas exitosamente en el laboratorio. Entre 1974 y 1982 el número de publicaciones disminuyó para incrementarse levemente entre 1983 y 1986 . Durante un período de nueve años, de 1987 a 1995, no se publicó ningún trabajo de descripción larvaria. Sólo últimamente se está observando un incremento en la cantidad de publicaciones sobre la morfología larvaria de decápodos (Fig. 1).

\section{Descripciones larvarias de decápodos chilenos por grupo taxonómico}

Penaeoidea y Caridea: No se ha descrito el desarrollo larvario de ninguna especie de la superfamilia Penaeoidea de aguas chilenas. Dentro de los camarones carídeos marinos (Tabla 1), la única descripción completa corresponde a Nauticaris magellanica (Wehrtmann y Albornoz, en prensa). El estudio sobre Chorismus antarcticus es un trabajo basado en larvas provenientes del plancton, y por lo tanto no es posible asegurar que las descripciones hechas por Gurney (1937) comprendan realmente los estadíos larvales de esta especie. Con respecto a especies de aguas continentales, Báez et al. (1983-84) describieron el desarrollo larval del camarón de río del norte, Cryphiops caementarius (Tabla 2). Los estadíos tempranos de la gran mayoría (93\%) de los carídeos representados en Chile no están decritos 
Tabla 1. Descripciones de larvas de decápodos marinos de aguas chilenas: Caridea y Thallassinidea; las abreviaciones ZI hasta ZXVII corresponden a zoea estadío 1 al 17.

Table 1. Description of marine decapod larvae from chilean waters: Caridea and Thallassinidea; abreviations ZI through ZXVII corresponds to the zoea stage 1 to 17.

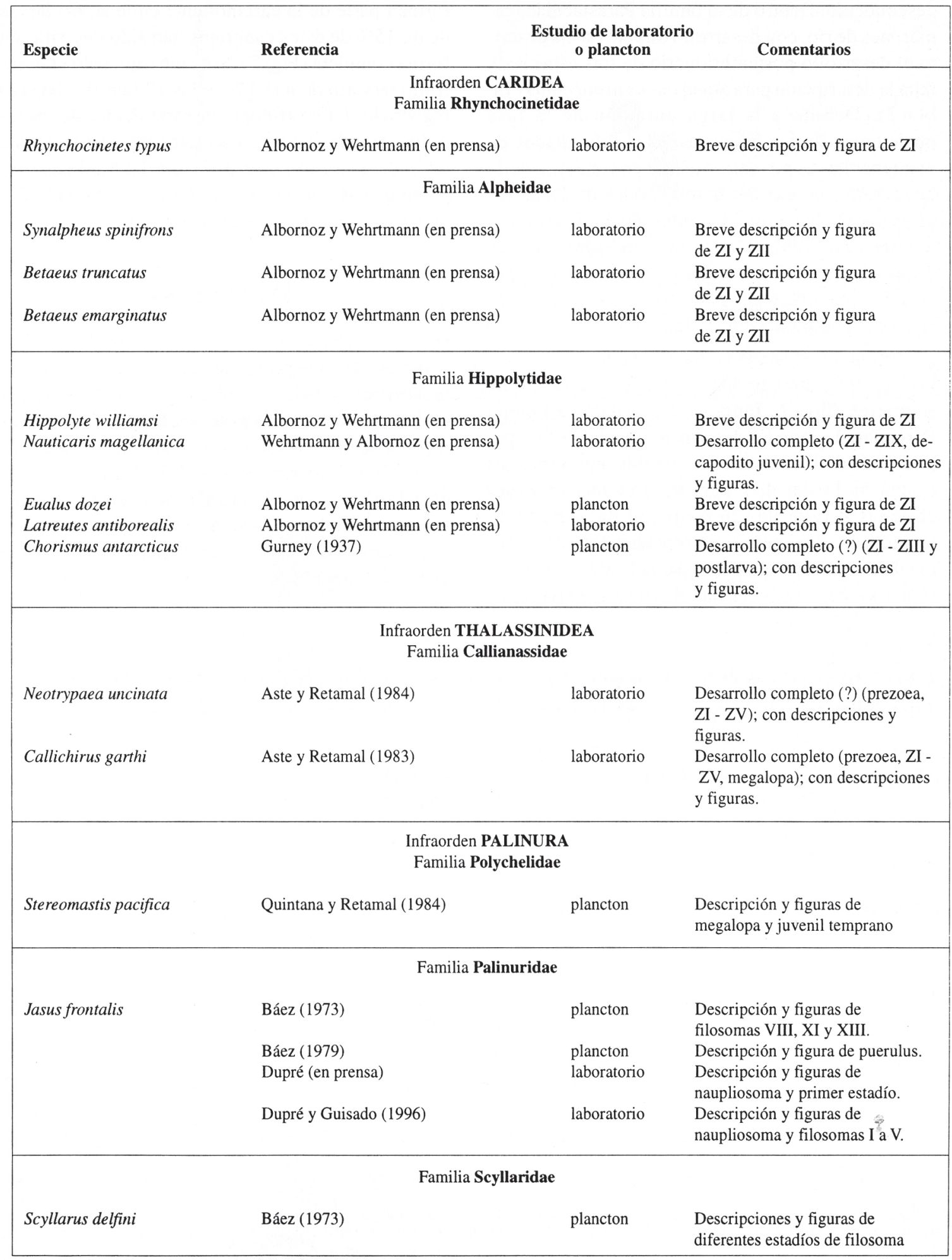


(Tabla 3), incluyendo Heterocarpus reedi, que es el camarón de importancia económica en Chile.

Astacidea y Thalassinidea: El desarrollo larvario de especies chilenas de la familia Nephropsidae es desconocido. Dentro de la familia Parastacidae (camarones de río, con desarrollo directo) se ha descrito el desarrollo postembrionario de tres especies y falta la descripción para Samastacus araucanius (Tabla 2). Debido a la larga duración de la fase planctónica de las langostas y las dificultades de mantener larvas por varios meses en condiciones de laboratorio, no existen descripciones de todos los estadíos de los representantes de Palinuridae y Scyllaridae (Tabla 1). De las cinco familias de Thalassinidea, sólo las larvas pertenecientes a especies de la familia Callianassidae han sido descritas en forma completa (Fig. 2, Tabla $3)$.

Anomura: Hasta la fecha, se conoce el desarrollo larvario completo de un total de 11 especies de anomuros (Fig. 2 y Tabla 4). Se destaca la familia Porcellanidae con las descripciones de cuatro especies y la familia Albuneidae con dos representantes (Tabla 5). De las diez familias presentes en aguas chilenas, existe una descripción del desarrollo larvario de, a lo menos, un representante de la mitad de las familias (Fig. 2). Llama la atención la ausencia de descripciones larvales para la mayoría de las especies de las familias Lithodidae y Galatheidae (Tablas 4 y 5), a pesar que varias especies son de importancia económica para el país.

Brachyura: Considerando todas las especies de Brachyura que forman parte de la carcinofauna chilena, las larvas de un $15 \%$ de estos cangrejos han sido descritas en forma completa (Fig. 2). Sin embargo, sólo el desarrollo larvario de un $41 \%$ de las 17 familias ha sido registrado. La morfología larvaria dentro de las familias grandes ha sido escasamente documentada, tal vez con la excepción de la familia Majidae, grupo en que se conoce el desarrollo larvario del $25 \%$ de las especies presentes (Tablas 4 y 6).

\section{CONCLUSIONES}

Hasta la fecha se ha descrito el desarrollo larvario completo de 31 especies de la carcinofauna chilena, lo que corresponde a un $13,4 \%$ del total de las especies presentes en el país (excluyendo las familias con desarrollo directo). La falta de disponibilidad de información similar resumida, dificulta la comparación de estos datos con aquéllos procedentes de otros países. Sin embargo, no existe duda que hasta la fecha, la morfología larvaria de los decápodos de Chile está pobremente documentada.

Tabla 2. Descripciones de larvas y estadíos tempranos de decápodos de aguas continentales de Chile. Table 2. Descriptions of larvae and early stages of decapods from continental waters of Chile.

\begin{tabular}{|c|c|c|c|}
\hline \multicolumn{4}{|c|}{$\begin{array}{l}\text { Estudio de laboratorio } \\
\text { o plancton }\end{array}$} \\
\hline \multicolumn{4}{|c|}{$\begin{array}{l}\text { Infraorden CARIDEA } \\
\text { Familia Palaemonidae }\end{array}$} \\
\hline \multirow[t]{2}{*}{ Cryphiops caementarius } & Norambuena (1977) & laboratorio & $\begin{array}{l}\text { Muy breve descripción de ZI y ZII, } \\
\text { sin figuras. }\end{array}$ \\
\hline & Báez et al. (1983-84) & laboratorio & $\begin{array}{l}\text { Desarrollo completo (ZI - ZXVII, } \\
\text { megalopa); con descripcionnes y figuras. }\end{array}$ \\
\hline \multicolumn{4}{|c|}{$\begin{array}{l}\text { Infraorden ASTACIDEA } \\
\text { Familia Parastacidae }\end{array}$} \\
\hline Parastacus pugnax & Rudolph y Ríos (1987) & laboratorio & $\begin{array}{l}\text { Descripción y figuras de los tres } \\
\text { primeros juveniles. }\end{array}$ \\
\hline Parastacus nicoleti & Rudolph y Zapata (1986) & laboratorio & $\begin{array}{l}\text { Descripción y figuras de dos } \\
\text { estadíos de postlarvas y el } \\
\text { primer juvenil. }\end{array}$ \\
\hline Samastacus spinifrons & Rudolph y Iracabal (1994) & laboratorio & $\begin{array}{l}\text { Descripción y figuras de los tres } \\
\text { primeros juveniles. }\end{array}$ \\
\hline
\end{tabular}




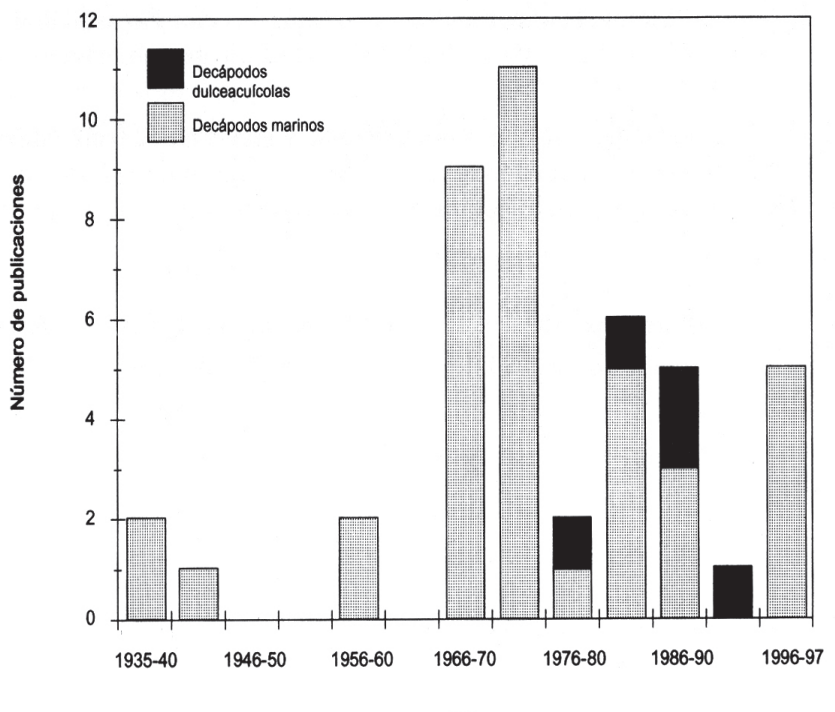

Años

Figura 1. Larvas de decápodos de aguas chilenas; número de descripciones publicadas entre 1935 y el presente. Figure 1. Decapod larvae in Chilean waters: number of descriptions published between 1935 and now.

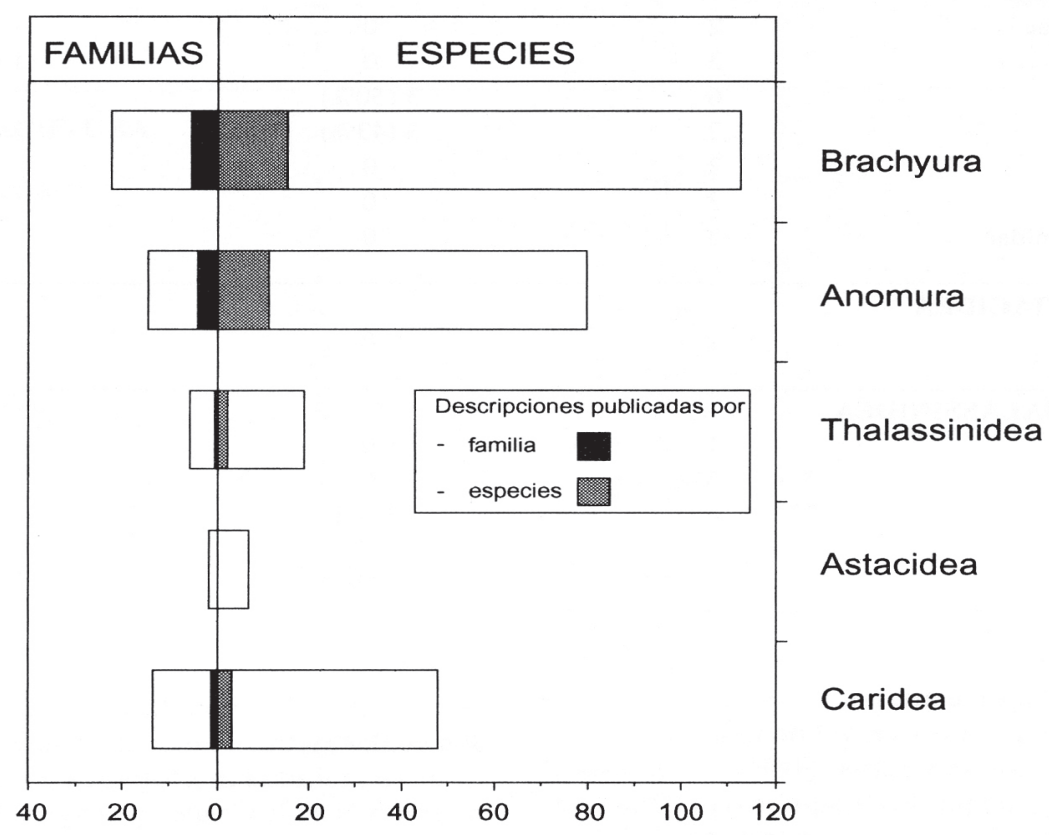

Número de familias/especies por Infraorden

Figura 2. Larvas de decápodos de aguas chilenas: número de descripciones publicadas en relación al número de familias y especies de cada infraorden.

Figure 2. Decapod larvae in Chilean waters: relation between number of published descriptions and the number of both families and species for each infraorder. 
Tabla 3. Crustáceos decápodos Penaeoidea, Caridea, Astacidea y Thalassinidea que viven en aguas de Chile. Número de especies por familia (excluidas aquéllas con desarrollo directo: Parastacidae y Aeglidae) y número de descripciones de larvas que se han publicado. Se indica el porcentaje de publicaciones con respecto al número de especies.

Table 3. Decapod crustacea Penaeoidea, Caridea, Astacidea y Thalassinidea from chilean waters. Number of species per family (excluding those whith direct development: Parastacidae and Aeglidae) and number of published descriptions of larvae. The percentage of publications relative to the number of species is indicated.

\begin{tabular}{|c|c|c|c|}
\hline $\begin{array}{l}\text { Familias por grupo } \\
\text { taxonómico }\end{array}$ & $\begin{array}{l}\text { Número de especies } \\
\text { reportadas para Chile }\end{array}$ & $\begin{array}{l}\text { Descripciones parciales } \\
\text { del desarrollo larvario: } \\
\text { número y porcentaje }\end{array}$ & $\begin{array}{l}\text { Descripciones completas } \\
\text { del desarrollo larvario: } \\
\text { número y porcentaje }\end{array}$ \\
\hline \multicolumn{4}{|c|}{ Infraorden PENAEOIDEA } \\
\hline Solenoceridae & 1 & 0 & 0 \\
\hline Aristeidae & 2 & 0 & 0 \\
\hline \multicolumn{4}{|l|}{ Infraorden CARIDEA } \\
\hline Pasiphaeidae & 3 & 0 & 0 \\
\hline Disciadidae & 1 & 0 & 0 \\
\hline Oplophoridae & 9 & 0 & 0 \\
\hline Nematocarcinidae & 2 & 0 & 0 \\
\hline Rhynchocinetidae & 1 & $1(100 \%)$ & 0 \\
\hline Campylonotidae & 2 & 0 & 0 \\
\hline Palaemonidae & 3 & 0 & $1(33 \%)$ \\
\hline Alpheidae & 6 & $3(50 \%)$ & 0 \\
\hline Hippolytidae & 7 & $3(43 \%)$ & $2(29 \%)$ \\
\hline Pandalidae & 3 & 0 & 0 \\
\hline Crangonidae & 3 & 0 & 0 \\
\hline Glyphocrangonidae & 3 & 0 & 0 \\
\hline \multicolumn{4}{|c|}{ Infraorden ASTACIDEA } \\
\hline Nephropsidae & 2 & 0 & 0 \\
\hline \multicolumn{4}{|c|}{ Infraorden THALASSINIDEA } \\
\hline Axiidae & 1 & 0 & 0 \\
\hline Callianassidae & 3 & 0 & $2(67 \%)$ \\
\hline Polychelidae & 6 & $1(17 \%)$ & 0 \\
\hline Palinuridae & 3 & $1(33 \%)$ & 0 \\
\hline Scyllaridae & 3 & $1(33 \%)$ & 0 \\
\hline
\end{tabular}

El grupo mejor estudiado, con descripciones larvales completas tanto a nivel de familia como de especie es el de los anomuros (50\% y 16\%, respectivamente), seguido por los braquiuros ( $41 \%$ y $15 \%$, respectivamente). La morfología larvaria de los camarones carídeos representados por 43 especies, está documentada por la única descripción de Nauticaris magellanica (Wehrtmann y Albornoz, en prensa). Este escaso conocimiento podría estar relacionado con dos factores: (1) El desarrollo larvario de los camarones carídeos comprende un número elevado de estadíos, dificultando su mantención en cultivos, los cuales frecuentemente demuestran además una gran variabilidad morfológica por estadío (Sandifer y Smith, 1979; Criales y Anger, 1986; Villamar y Brusca, 1988; Wehrtmann y Albornoz, en prensa). En consecuencia, existen múltiples y grandes dificultades para elaborar una descripción larvaria completa en un carídeo (Wehrtmann, obs. pers.), lo que explica el escaso número de publicaciones sobre este grupo de crustáceos. (2) Solamente una especie (Heterocarpus reedi) juega un rol importante en la 
Tabla 4. Decápodos anomuros y braquiuros: número de especies por familia reportadas para Chile y número de descripciones de larvas que se han publicado. Se indica el porcentaje de publicaciones con respecto al número de especies.

Table 4. Anomuran and brachiuran decapods: number of species per family reported for Chile and number of publications of larvae descriptions. The percentage of publications relative to the number of species is indicated.

\begin{tabular}{|c|c|c|c|}
\hline Grupo taxonómico & Número de especies & $\begin{array}{l}\text { Número de } \\
\text { descripciones } \\
\text { larvales parciales }(\%)\end{array}$ & $\begin{array}{l}\text { Número de } \\
\text { descripciones } \\
\text { larvales completas }(\%)\end{array}$ \\
\hline \multicolumn{4}{|c|}{ Infraorden ANOMURA } \\
\hline Coenobitidae & 1 & 0 & 0 \\
\hline Diogenidae & 3 & 0 & 0 \\
\hline Lithodidae & 16 & 0 & $2(13 \%)$ \\
\hline Paguridae & 6 & 0 & 0 \\
\hline Parapaguridae & 4 & 0 & 0 \\
\hline Chirostylidae & 2 & 0 & 0 \\
\hline Galatheidae & 17 & $1(6 \%)$ & $2(12 \%)$ \\
\hline Porcellanidae & 15 & 0 & $4(27 \%)$ \\
\hline Albuneidae & 2 & 0 & $2(100 \%)$ \\
\hline Hippidae & 2 & 0 & $1(50 \%)$ \\
\hline \multicolumn{4}{|c|}{ Infraorden BRACHYURA } \\
\hline Homolodromidae & 1 & 0 & 0 \\
\hline Dromidae & 1 & 0 & 0 \\
\hline Homolidae & 1 & 0 & 0 \\
\hline Calappidae & 2 & $1(50 \%)$ & 0 \\
\hline Majidae & 16 & 0 & $4(25 \%)$ \\
\hline Hymenosomidae & 1 & 0 & $1(100 \%)$ \\
\hline Atelecyclidae & 2 & 0 & 0 \\
\hline Cancridae & 4 & $2(50 \%)$ & $2(50 \%)$ \\
\hline Corystidae & 2 & 0 & 0 \\
\hline Bellidae & 5 & 0 & $3(60 \%)$ \\
\hline Geryonidae & 1 & 0 & 0 \\
\hline Portunidae & 7 & $1(14 \%)$ & 0 \\
\hline Platyxanthidae & 2 & 0 & 0 \\
\hline Xanthidae & 23 & $1(4 \%)$ & $2(9 \%)$ \\
\hline Grapsidae & 18 & $3(17 \%)$ & $2(11 \%)$ \\
\hline Pinnotheridae & 8 & $1(13 \%)$ & $1(13 \%)$ \\
\hline Ocypodidae & 3 & 0 & 0 \\
\hline
\end{tabular}


Tabla 5. Larvas de decápodos marinos de aguas chilenas del Infraorden Anomura presentes en aguas chilenas: descripciones publicadas.

Table 5. Infraorder Anomura marine decapod larvae present in chilean waters: published descriptions.

\begin{tabular}{|c|c|c|c|}
\hline Especie & \multicolumn{3}{|c|}{$\begin{array}{l}\text { Estudio de laboratorio } \\
\text { o plancton }\end{array}$} \\
\hline \multicolumn{4}{|c|}{ Familia Lithodidae } \\
\hline Lithodes santolla & Campodonico (1971) & laboratorio & $\begin{array}{l}\text { Desarrollo completo (ZI - ZII, } \\
\text { glaucothoe); con descripciones } \\
\text { y figuras. }\end{array}$ \\
\hline Paralomis granulosa & Campodonico y Guzmán (1981) & laboratorio & $\begin{array}{l}\text { Desarrollo completo (ZI - ZII, } \\
\text { megalopa); con descripciones } \\
\text { y figuras. }\end{array}$ \\
\hline \multicolumn{4}{|c|}{ Familia Galatheidae } \\
\hline Pleuroncodes monodon & Fagetti y Campodonico (1971a) & laboratorio & $\begin{array}{l}\text { Desarrollo completo (ZI - ZV); con } \\
\text { descripciones y figuras. }\end{array}$ \\
\hline Munida subrugosa & Roberts (1973) & plancton & $\begin{array}{l}\text { Desarrollo completo (ZI - ZV, } \\
\text { megalopa); con descripciones y figuras. }\end{array}$ \\
\hline Cervimunida johni & Fagetti (1960b) & laboratorio & Descripción y figuras de ZI \\
\hline \multicolumn{4}{|c|}{ Familia Porcellanidae } \\
\hline Petrolisthes granulosus & Saelzer et al. (1986) & laboratorio & $\begin{array}{l}\text { Desarrollo completo (prezoea, ZI - } \\
\text { ZII, megalopa); con descripciones } \\
\text { y figuras. }\end{array}$ \\
\hline Petrolisthes laevigatus & Albornoz y Wehrtmann (1996) & laboratorio & $\begin{array}{l}\text { Desarrollo completo (prezoea, ZI - } \\
\text { ZII, megalopa, primer cangrejo); con } \\
\text { descripciones y figuras. }\end{array}$ \\
\hline Petrolisthes violaceus & Wehrtmann et al. (1997) & laboratorio & $\begin{array}{l}\text { Desarrollo completo (prezoea, ZI - } \\
\text { ZII, megalopa); con descripciones } \\
\text { y figuras. }\end{array}$ \\
\hline Allopetrolisthes angulosus & Wehrtmann et al. (1996) & laboratorio & $\begin{array}{l}\text { Desarrollo completo (prezoea, ZI - } \\
\text { ZII, megalopa, primer cangrejo); } \\
\text { con descripciones y figuras. }\end{array}$ \\
\hline \multicolumn{4}{|c|}{ Familia Albuneidae } \\
\hline Lepidopa chilensis & Sánchez y Aguilar (1975) & laboratorio & $\begin{array}{l}\text { Desarrollo completo (ZI - ZV; } \\
\text { megalopa); con descripciones } \\
\text { y figuras). }\end{array}$ \\
\hline Blepharipoda spinimana & Knight (1968) & plancton & $\begin{array}{l}\text { Desarrollo completo ? (ZI - ZIV); } \\
\text { con descripciones y figuras. }\end{array}$ \\
\hline \multicolumn{4}{|c|}{ Familia Hippidae } \\
\hline Emerita analoga & Johnson y Lewis (1942) & plancton & $\begin{array}{l}\text { Desarrollo completo ? (ZI - ZV); } \\
\text { con descripciones y figuras. }\end{array}$ \\
\hline
\end{tabular}


Tabla 6. Larvas de decápodos marinos de aguas chilenas del Infraorden Brachyura: descripciones publicadas. Table 6. Infraorder Brachyura marine decapod larvae present in chilean waters: published descriptions.

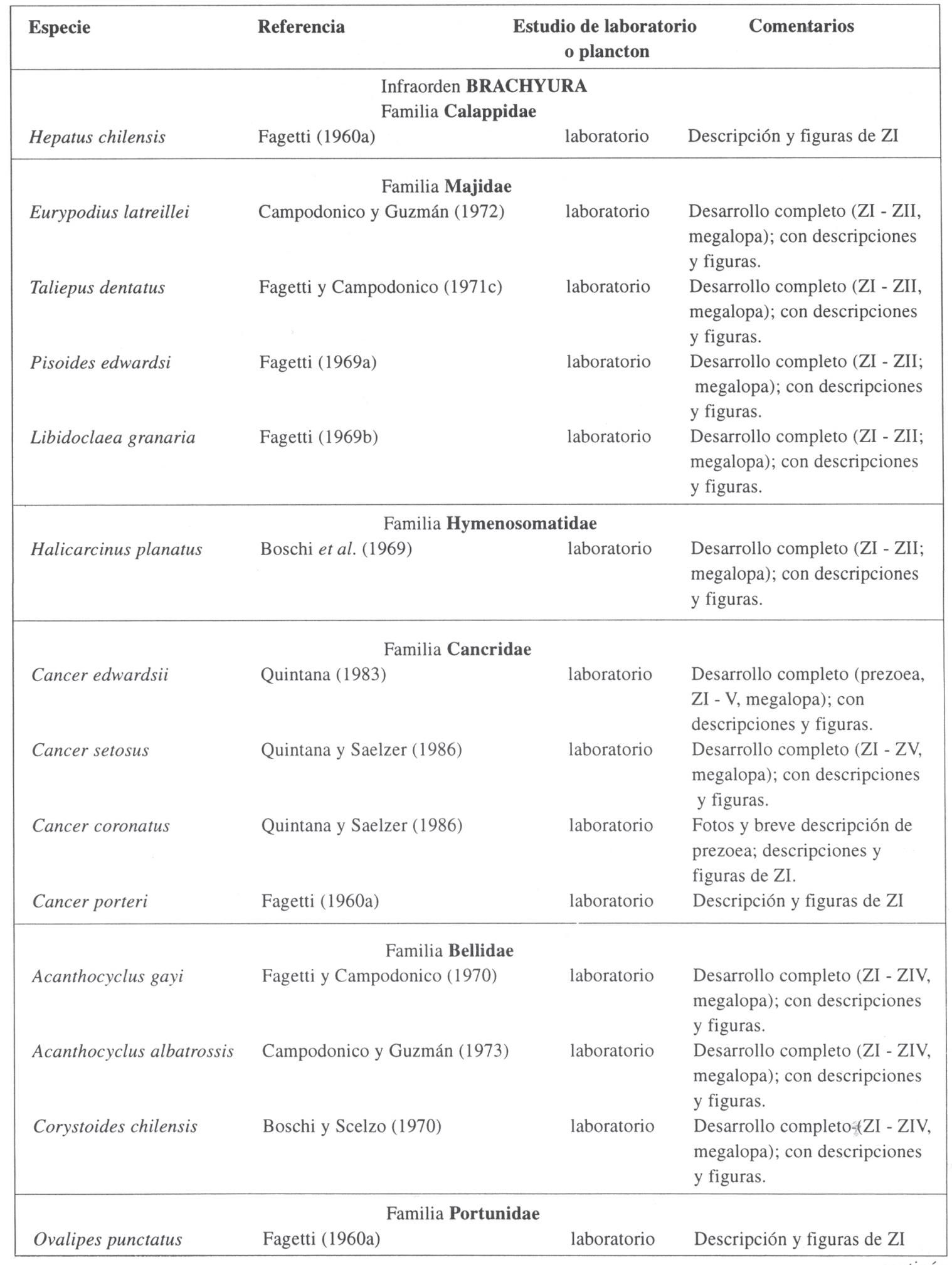




\begin{tabular}{|c|c|c|c|}
\hline \multicolumn{4}{|c|}{ Familia Xanthidae } \\
\hline Homalaspis plana & Fagetti (1960a) & laboratorio & Descripción y figuras de ZI \\
\hline & Fagetti (1970) & laboratorio & $\begin{array}{l}\text { Desarrollo completo (ZI - ZIV, } \\
\text { megalopa); con descripciones } \\
\text { y figuras. }\end{array}$ \\
\hline Pilumnoides perlatus & Fagetti y Campodonico (1973) & laboratorio & $\begin{array}{l}\text { Desarrollo completo (ZI - ZV; } \\
\text { megalopa); con descripciones } \\
\text { y figuras. }\end{array}$ \\
\hline \multicolumn{4}{|c|}{ Familia Grapsidae } \\
\hline Leptograpsus variegatus & Wear (1970) & laboratorio & $\begin{array}{l}\text { Descripción breve de la } \\
\text { prezoea; descripción y figuras } \\
\text { de ZI. }\end{array}$ \\
\hline Hemigrapsus crenulatus & Wear (1970) & laboratorio & $\begin{array}{l}\text { Descripción breve de la } \\
\text { prezoea; descripción y figuras } \\
\text { de Zl. }\end{array}$ \\
\hline Cyclograpsus cinereus & Costlow y Fagetti (1967) & laboratorio & $\begin{array}{l}\text { Desarrollo completo (ZI - ZV, } \\
\text { megalopa); con descripciones } \\
\text { y figuras. }\end{array}$ \\
\hline Cyclograpsus punctatus & Fagetti y Campodonico (1971b) & laboratorio & $\begin{array}{l}\text { Desarrollo completo ( } \mathrm{ZI}-\mathrm{ZV} \text {, } \\
\text { megalopa); con descripciones } \\
\text { y figuras. }\end{array}$ \\
\hline Plagusia chabrus & Wear (1970) & laboratorio & $\begin{array}{l}\text { Descripción breve de la } \\
\text { prezoea; descripción y figuras } \\
\text { de ZI. }\end{array}$ \\
\hline \multicolumn{4}{|c|}{ Familia Pinnotheridae } \\
\hline Pinnotheres politus & Saelzer y Hapette (1986) & laboratorio & $\begin{array}{l}\text { Desarrollo completo (ZI - ZV, } \\
\text { megalopa); con descripciones } \\
\text { y figuras. }\end{array}$ \\
\hline Pinnaxodes chilensis & Schwabe (1936) & laboratorio & Descripción y figuras de ZI \\
\hline & Gutiérrez-Martínez (1971) & laboratorio & Descripción y figuras de ZI \\
\hline
\end{tabular}

pesquería de decápodos en Chile, mientras que la mayoría de las otras especies son de pequeño tamaño y/o baja densidad (Holthuis, 1952). La escasa importancia económica de este grupo de decápodos ha dificultado probablemente la obtención de fondos para el estudio de su desarrollo larval y, en consecuencia, ha disminuido el interés de los científicos por este tipo de investigaciones.

El adecuado manejo de la pesquería de decápodos requiere el conocimiento de la morfología larvaria para poder elucidar las relaciones entre «stock» y reclutamiento, que es uno de los problemas críticos para el manejo de la pesca (Caddy, 1989). En este sentido, la falta de descripciones larvales para especies de importancia económica, como Heterocarpus reedi, Cancer coronatus y $C$. porteri, se debería considerar como grave, dificultando no sólo la comprensión de la biología de estas especies, sino también el manejo adecuado de estos recursos.

\section{AGRADECIMIENTOS}

Los autores agradecen al Dr. Enrique Boschi (INIDEP, Argentina), al Biólogo Marino Italo Campodonico (Subsecretaría de Pesca, Chile) y al Dr. Jaime Mendo A. (Universidad Nacional Agraria La Molina, Perú) por su colaboración en la recopilación de la literatura. Queremos agradecer en forma especial al Biólogo Marino E. Alejandro Abarca M. por el envío de valiosa información acerca de descripciones larvales de los decápodos chi- 
lenos. El primer autor agradece al «Servicio Alemán de Intercambio Académico» (DAAD) y a la «Gesellschaft für Technische Zusammenarbeit» (GTZ) por la oportunidad brindada de trabajar cinco años como profesor huésped en el Instituto de Zoología de la Universidad Austral de Chile.

\section{REFERENCIAS}

Albornoz, L. y I.S. Wehrtmann. 1996. Aspects of the reproductive biology of Petrolisthes laevigatus (Guérin, 1835) (Decapoda: Anomura: Porcellanidae). II. Complete larval development under laboratory conditions. Arch. Fish. Mar. Res., 43(2): 137-157.

Anger, K. 1995. The conquest of freshwater and land by marine crabs: adaptations in life-history patterns and larval energetics. J. Exp. Mar. Biol. Ecol., 193: 119-145.

Anger, K., E. Spivak, C. Bas, D. Ismael y T. Luppi. 1994. Hatching rhythms and dispersion of decapod crustacean larvae in a brackish coastal lagoon in Argentina. Helgoländer Meeresunters, 48: 445-466.

Aste, A. y M.A. Retamal. 1983. Desarrollo larval de Callianassa garthi Retamal, 1975 bajo condiciones de laboratorio. Cienc. Tec. Mar, CONA, 7: 5-26.

Aste, A. y M.A. Retamal. 1984. Desarrollo larval de Callianassa uncinata H. Milne Edwards, 1837 (Decapoda, Callianassidae) bajo condiciones de laboratorio. Gayana, Zool., 48(1-2): 4156.

Báez, R.P. 1973. Larvas phyllosoma del Pacífico sur oriental (Crustacea, Macrura, Scyllaridae). Rev. Biol. Mar., Valparaíso, 15(1): 115-130.

Báez, R.P. 1979. El puerulus de Jasus frontalis (H. Milne Edwards, 1837) (Crustacea: Decapoda: Palinuridae). Rev. Biol. Mar. Dep. Oceanol. Univ. Chile, 16(3): 225-228.

Báez, R.P. 1995. Crustáceos. In: J.A. Simonetti, M.T.K. Arroyo, A.E. Spotorno y E. Lozado (eds.), Diversidad Biológica de Chile. CONICYT, Santiago, pp. 189-194.

Báez, R.P., J. Sanzana D. y J. Weinborn. 19831984. Contribución al conocimiento de la morfología larvaria de Cryphiops caementarius, camarón de río del norte de Chile. Bol. Mus. Nac. Hist. Nat. Chile, 40: 153-172.
Boschi, E.E. y M.A. Scelzo. 1970. Desarrollo larval del cangrejo Corystoides chilensis Milne Edwards y Lucas en laboratorio (Decapoda, Brachyura, Atelecyclidae). Physis, 30(80): 113124.

Boschi, E.E., M.A. Scelzo y B. Goldstein. 1969. Desarrollo larval del cangrejo, Halicarcinus planatus (Fabricius) (Crustacea, Decapoda, Hymenosomidae), en el laboratorio, con observaciones sobre la distribución de la especie. Bull. Mar. Sci., 19(1): 225-242.

Bowman, T.E. y L.G. Abele. 1982. Classification of recent Crustacea. In: L.G. Abele (ed.) The Biology of Crustacea. Vol. 1. Systematics, the fossil record and biogeography. Academic Press, New York, pp. 1-27.

Brattström, H. y A. Johanssen. 1983. Ecological and regional zoogeography of the marine benthic fauna of Chile. Report No. 49 of the Lund University Chile Expedition 1948-49. Sarsia, 68: 289-339.

Caddy, J.F. 1989. Marine invertebrate fisheries. Their assessment and management. J. Wiley and Sons, Inc. New York. 752 pp.

Campodonico, G.I. 1971. Desarrollo larval de la centolla Lithodes antarctica Jacquinot en condiciones de laboratorio. Ans. Inst. Pat., Punta Arenas (Chile), 2(1-2): 181-190.

Campodonico, G.I. y L. Guzmán. 1972. Desarrollo larval de Eurypodius latreillei Guérin en condiciones de laboratorio. Ans. Inst. Pat., Punta Arenas (Chile), 3(1-2): 233-247.

Campodonico, G.I. y L. Guzmán. 1973. Contribución a la biología de Acanthocyclus albatrossis Rathbun 1898. Ans. Inst. Pat., Punta Arenas (Chile), IV(1-2): 373-416.

Campodonico, G.I. y L. Guzmán. 1981. Larval development of Paralomis granulosus (Jacquinot) under laboratory conditions (Decapoda, Anomura, Lithodidae). Crustaceana, 40(3): 272-285.

Costlow, J.D., Jr. y E. Fagetti. 1967. The larval development of the crab, Cyclograpsus cinereus Dana, under laboratory conditions. Pacific Sci., 21: 166-77.

Criales, M.M. y K. Anger. 1986. Experimental studies on the larval development of the shrimps Crangon crangon and C. allmanni. Helgoländer Meeresunters, 40: 241-265. 
Dupré, M.E. (en prensa). Primera phyllosoma de la langosta de Juan Fernández, Jasus frontalis. Rev. Chil. Hist. Nat., 60.

Dupré, E. y Ch. Guisado. 1996. Identificación de los primeros estados se phyllosoma de la langosta de Juan Fernández (Jasus frontalis) mantenidos en laboratorio. Invest. Mar. Valparaíso, 24: 39-50.

Fagetti, G.E. 1960. Primer estadio larval de cuatro crustáceos braquiuros de la Bahía de Valparaíso. Rev. Biol. Mar. Valparaíso, 10: 143-154.

Fagetti, G.E. 1960. Huevos y el primer estadio larval del langostino (Cervimunida johni Porter 1903). Rev. Chilena Hist. Nat., 55: 33-42.

Fagetti, G.E. 1969a. Larval development of the spider crab Pisoides edwardsi (Decapoda, Brachyura) under laboratory conditions. Mar. Biol., 4: 160-165.

Fagetti, G.E. 1969b. The larval development of the spider crab Libidoclaea granaria $\mathrm{H}$. Milne Edwards y Lucas under laboratory conditions (Decapoda Brachyura, Majidae, Pisinae). Crustaceana, 17(2): 131-140.

Fagetti, G.E. 1970. Desarrollo larval en el laboratorio de Homalaspis plana (Milne-Edwards) (Crustacea Brachyura; Xanthidae). Rev. Biol. Mar. Valparaíso, 14(2): 29-49.

Fagetti, G.E. y I. Campodonico. 1970. Desarrollo larval en el laboratorio de Acanthocyclus gayi Milne-Edwards et Lucas (Crustacea Brachyura; Atelecyclidae, Acanthocyclinae). Rev. Biol. Mar., Valparaíso, 14(2): 63-78.

Fagetti, G.E. y I. Campodonico. 1971a. Larval development of the red crab Pleuroncodes monodon (Decapoda Anomura: Galatheidae) under laboratory conditions. Mar. Biol., 8: 70-81.

Fagetti, G.E. y I. Campodonico. 1971b. The larval development of the crab Cyclograpsus punctatus H. Milne Edwards, under laboratory conditions (Decapoda Brachyura, Grapsidae, Sesarminae). Crustaceana 21(2): 183-195.

Fagetti, G.E. y I. Campodonico. 1971c. Desarrollo larval en el laboratorio de Taliepus dentatus (Milne-Edwards) (Crustacea Brachyura: Majidae, Acanthocyclinae). Rev. Biol. Mar., Valparaíso, 14(3): 1-14.

Fagetti, G.E. y I. Campodonico. 1973. Larval development of Pilumnoides perlatus (Brachyura: Xanthidae) under laboratory conditions. Mar. Biol., 18: 129-139.
Gurney, R. 1937. Larvae of decapod Crustacea. Part IV. Hippolytidae. Discovery Rep., 14: 351-404.

Gutiérrez-Martínez, J. 1971. Notas biológicas sobre Pinnoxodes chilensis (M. Edwards) y descripción de su primera zoea. Not. Mens. Mus. Nac. Hist. Nat., 15(176): 3-11.

Holthuis, L.B. 1952. The Crustacea Decapoda Macrura of Chile. Lund Univ. Arssk., (n.s. 2) 47(10): 1-109.

Ingle, R. 1992. Larval stages of northeastern Atlantic crabs. Chapman and Hall, London. 363 pp.

Jalihal, D.R., K.N. Sankolli y S. Shenoy. 1993. Evolution of larval developmental patterns and the process of freshwaterization in the prawn genus Macrobrachium Bate, 1868 (Decapoda, Palaemonidae). Crustaceana, 65: 365-376.

Johnson, M.W. y W.M. Lewis. 1942. Pelagic larval stages of the sand crabs Emerita analoga (Stimpson), Blepharipoda occidentalis Randall and Lepidopa myops Stimpson. Biol. Bull., 83: 67-87.

Knight, M.D. 1968. The larval development of Blepharipoda occidentalis Randall and $B$. spinimana (Philippi) (Decapoda, Albuneidae). Proc. Calif. Acad. Sci., 35(16): 337-370.

Lindley, J.A. 1987. Continuous plankton records: the geographical distribution and seasonal cycles of decapod crustacean larvae and pelagic postlarvae in the north-eastern Atlantic Ocean and the North Sea, 1981-3. J. Mar. Biol. Ass., U.K., 67: 145-167.

Lindley, J.A., R. Williams y H.G. Hunt. 1993. Anomalous seasonal cycles of decapod crustacean larvae in the North Sea plankton in an abnormmaly warm year. J. Exp. Mar. Biol. Ecol., 172: 47-67.

Manning, R.B. y L.B. Holthuis. 1989. Two new genera and nine species of geryonid crabs (Crustacea, Decapoda, Geryonidae). Proc. Biol. Soc. Wash., 102(1): 50-77.

Morgan, S.G. 1990. Impact of planktivorous fishes on dispersal, hatching, and morphology of estuarine crab larvae. Ecology, 71: 1639-1652.

Morgan, S.G. 1992. Predation by planktonic and benthic invertebrates on larvae of estuarine crabs. J. Exp. Mar. Biol. Ecol., 163: 91-110.

Norambuena, C.R. 1977. Antecedentes biológicos de Cryphiops caementarius (Mol, 1782) en el 
estero «El Culebrón» (Crustacea, Decapoda, Palaemonidae). Biol. Pesq. Chile, 9: 7-19.

Palma, S. 1976. Meroplancton de la región de Valparaíso. Cienc. Tec. Mar, CONA, 2: 99-116.

Palma, S. 1980. Larvas de crustáceos decápodos capturadas frente a la costa de Valparaíso. Invest. Mar., Valparaíso, 8: 129-144.

Palma, S. 1994. Distribución y abundancia de larvas de langostino colorado Pleuroncodes monodon frente a la costa de Concepción, Chile. Invest. Mar., Valparaíso, 22: 13-29.

Palma, S. y P. Arana. 1997. Aspectos reproductivos del langostino colorado (Pleuroncodes monodon H. Milne Edwards, 1837), frente a la costa de Concepción, Chile. Invest. Mar., Valparaíso, 25: 203-221.

Palma, S., J. Meruane y A. Mujica. 1976. Observaciones sobre el meroplancton del Archipiélago de Juan Fernández. Enero 1974. Cienc. Tec. Mar, CONA, 2: 117-126.

Perkins, H.C. 1973. The larval stages of the deep sea red crab, Geryon quinquedens Smith, reared under laboratory conditions (Decapoda: Brachyrhyncha). US Fish. Bull., 71(1): 69-82.

Quintana, R. 1983. Larval development of the edible crab, Cancer edwardsi Bell, 1835 under laboratory conditions (Decapoda, Brachyura). Rep. Usa Mar. Biol. Inst. Kochi Univ., 5: 1-19.

Quintana, R. y M.A. Retamal. 1984. Two pelagic stages of Stereomastis pacifica (Faxon) collected in the Chile-Peru trench (Polychelidae: Macrura). Res. Crust., 13-14: 133-142.

Quintana, R. y H. Saelzer. 1986. The complete larval development of the edible crab, Cancer setosus Molina and observations on the prezoeal and first zoeal stages of $C$. coronatus Molina (Decapoda: Brachyura, Cancridae). J. Fac. Sci. Hokkaido Univ. Ser. VI Zool., 24(40): 267-303.

Retamal, M.A. 1981. Catálogo ilustrado de los crustáceos decápodos de Chile. Gayana, Zool., 44: 7-110.

Rice, A.L. 1980. Crab zoeal morphology and its bearing on the classification of the Brachyura. Trans. Zool. Soc., London, 35: 271-424.

Rice, A.L. y R.W. Ingle. 1975. A comparative study of the larval morphology of the British portunid crabs Macropipus puber (L.) and M. holsatus (Fabricius), with a discussion of generic and subfamilial larval characters within the Portunidae. Bull. Brit.Mus. (Nat. Hist.), Zool., 28(4): 123 151.

Roberts, P.E. 1973. Larvae of Munida subrugosa (White), 1847, from Perseverance Harbour, Campbell Island. J. Roy. Soc. New Zealand, 3(3): 393-408.

Rudolph, E. y J.C. Iracabal. 1994. Desarrollo embrionario y postembrionario del camarón de río Samastacus spinifrons (Philippi, 1882) (Decapoda, Parastacidae), en condiciones de laboratorio. Bol. Soc. Biol. Concepción, 65: 4349.

Rudolph, E. y J. Ríos. 1987. Desarrollo ontogénetico del camarón de las vegas Parastacus pugnax (Poeppig, 1835), en condiciones de laboratorio. Biota, 3: 45-58.

Rudolph, E. y L. Zapata. 1986. Desarrollo embrionario y postlarval del camarón de las vegas Parastacus nicoleti (Philippi, 1882), en condiciones de laboratorio. Biota, 2: 37-50.

Saelzer, H.E. y A.M. Hapette. 1986. Desarrollo larvario de Pinnotheres politus (Smith, 1870) (Brachyura, Pinnotheridae) en condiones de laboratorio. Gayana, Zool., 50(1-4): 63-79.

Saelzer, H.E., R. Quintana y R. Quiñones. 1986. Larval development of Petrolisthes granulosus (Guérin, 1835) (Decapoda: Anomura: Porcellanidae) under laboratory conditions. J. Crust. Biol., 6(4): 804-819.

Sánchez, G. y P. Aguilar. 1975. Notas sobre crustáceos del mar peruano. I. Desarrollo larvario de Lepidopa chilensis Lenz (Decapoda, Anomura: Albunidae). An. Cient. Univ. Nac. Agraria La Molina, 12(1-2): 1-12.

Sandifer, P.A. y T.I.J. Smith. 1979. Possible significance of variation in the larval development of palaemonid shrimp. J. Exp. Mar. Biol. Ecol., 39: 55-64.

Schwabe, G.H. 1936. Investigaciones sobre Loxechinus albus Mol. y Pinnotheres chilensis Edw. Bol. Soc. Biol. Concepción, 19(2): 125-136.

Villamar, D.F. y G.J. Brusca. 1988. Variation in the larval development of Crangon nigricauda (Decapoda: Caridea), with notes on larval morphology and behavior. J. Crust. Biol., 8: 410419. 
Wear, R.G. 1970. Life-history studies on New Zealand Brachyura. N.Z. J. Mar. Freswat. Res., 4(1): 3-35.

Wehrtmann, I.S. 1989. Seasonal occurrence and abundance of caridean shrimp larvae at Helgoland, German Bight. Helgoländer Meeresunters, 43: 87-112.

Wehrtmann, I.S. 1994. Larval production of the caridean shrimp, Crangon septemspinosa, in waters adjacent to Chesapeake Bay, USA, in relation to oceanographic conditions. Estuaries, 17(2): 508-517.

Wehrtmann, I.S. y L. Albornoz. (en prensa). Larval development of Nauticaris magellanica (A. Milne Edwards, 1891) (Decapoda: Caridea: Hippolytidae), reared under laboratory conditions. Bull. Mar. Sci., 60(2):

Recibido el 18 de junio de 1996.

Aceptado el 16 de octubre de 1996.
Wehrtmann, I.S., L. Albornoz, L.M. Pardo y D. Véliz. (1997). The larval developmental of Petrolisthes violaceus (Guérin, 1831) (Decapoda, Anomura, Porcellanidae) from Chilean waters, cultivated under laboratory conditions. Crustaceana, 70(5): 562-583.

Wehrtmann, I.S., L. Albornoz, D. Véliz y L.M. Pardo. (1996). Description of the early developmental stages, including the first crab, of Allopetrolisthes angulosus (Guérin, 1835) (Decapoda: Anomura: Porcellanidae) from Chilean waters, cultivated under laboratory conditions. J. Crust. Biol., 16(4): 730-747.

Wehrtmann, I.S. y W. Greve. 1995. Larvae of uncommon caridean decapods in the German Bight: species composition, distribution and abundance. Helgoländer Meeresunters, 49: 867-886. 